\title{
BIOMASSA MICROBIANA E SUA ATIVIDADE EM SOLOS SOB DIFERENTES SISTEMAS DE PREPARO E SUCESSÃO DE CULTURAS ${ }^{(1)}$
}

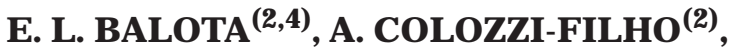 \\ D. S. ANDRADE ${ }^{(2)} \&$ M. HUNGRIA $(3,4)$
}

\begin{abstract}
RESUMO
Foi avaliada a biomassa microbiana e sua atividade, em solo submetido às sucessões de culturas trigo/soja e trigo/milho, preparado pelo sistema convencional e em plantio direto. A avaliação foi realizada em um experimento realizado em um Latossolo R oxo desde 1976 na Estação Experimental do Instituto Agronômico do Paraná-IAPAR, em Londrina (PR). Foram coletadas amostras na profundidade de 0-15 cm, dez dias após o plantio e sete dias antes da colheita da cultura de verão e de inverno dos anos de 1992, 1993 e 1994. Avaliaram-se a respiracão basal do solo e o carbono da biomassa microbiana pelo método de fumigação-incubação; o nitrogênio da biomassa microbiana, pelo método da fumi gação-extração, o quociente metabólico e a relação $C_{\text {mid }} / C_{\text {org }}$ dos solos. Houve poucas diferenças significativas nos parâmetros avaliados, em função das diferentes sucessões de culturas. As parcelas sob plantio direto apresentaram incrementos de 118 e 101\% no carbono e nitrogênio da biomassa microbiana, respectivamente, de $73 \%$ na respiração basal e de $96 \%$ na relação $C_{\text {mic }} / C_{\text {org }}$, enquanto houve um decrésci mo de $28 \%$ no quociente metabólico (qCO2). Os dados obtidos evidenciam que a prática do plantio direto proporciona maior biomassa microbiana e menor perda relativa de $\mathrm{C}$ via respiração, podendo determinar, assim, maior acúmulo de C no solo a longo prazo. Conseqüentemente, os parâmetros microbiológicos mostraram-se bons indicadores de alterações do solo em função do manejo.
\end{abstract}

Termos de indexação: carbono orgânico, plantio direto, plantio convencional, quociente metabólico, relação $\mathrm{C}_{\text {mic }} / \mathrm{C}_{\text {org }}$.

(1) Trabalho apresentado no XXVI Congresso Brasileiro de Ciência do Solo, realizado no Rio de J aneiro (RJ ), em julho de 1997. Recebido para publicação em setembro de 1997 e aprovado em setembro de 1998.

(2) Pesquisador da Área de Solos, Instituto Agronômico do Paraná-I APAR. Caixa Postal 481, CEP 86001-970 Londrina (PR).

(3) Pesquisadora da Embrapa/CNPSo. Caixa Postal 231, CEP 86001-970 Londrina (PR).

(4) Bolsista do CNPq. 


\title{
SUMMARY: MICROBIAL BIOMASS AND ITS ACTIVITY IN SOILS UNDER DIFFERENT TILLAGE AND CROP ROTATION SYSTEMS
}

\begin{abstract}
In this study, microbial biomass and its activity were evaluated on a soil submitted to crop rotations with wheat/ soybean and wheat/ maize under theno-tillageand conventional tillage systems. The evaluation was performed on an experiment established since 1976 at theE xperimental Station of I nstitutoAgronômi co do Paraná (IAPAR), Londrina (PR), Brazil. Soil samples were taken in the plough layer $(0-15 \mathrm{~cm})$ ten days after planting and seven days before harvesting summer and winter crops during 1992, 1993 and 1994. The basal respiration and themi crobial bi omass carbon wereevaluated by thefumi gation-incubation method, and the microbial biomass nitrogen was evaluated by fumigation-extraction, the determination of the metabolic coefficient and therelation $\mathrm{C}_{\text {mid }} \mathrm{C}_{\text {org }}$ of the soil. There were few significant differences on the parameters related above as a result of the different crop rotations. However, when compared with the conventional tillage the plots under the notillage system have shown increases of 118 and $101 \%$ on carbon and nitrogen microbial biomass, respectively, of $73 \%$ on basal respiration and of $96 \%$ on the relation $\mathrm{C}_{\text {mid }} \mathrm{C}_{\text {org, }}$ while the metabolic quotient $\left(\mathrm{qCO}_{2}\right)$ has decreased by $28 \%$. The continuous use of the notillagesystem, either under wheat/ soybean or wheat/ maizecrop rotation resulted in increases in microbial bi omass and decreases in mi crobial respiration, ther fore having measurable Iong term effects on the increase of soil $\mathrm{C}$ content. Consequently, microbial biomass has shown to bea good indicator to evaluatetheeffects of long term management on soil al teration.
\end{abstract}

Index terms: organic carbon, no-tillage, conventional tillage, metabolic quotient, ratio $\mathrm{C}_{\text {mid }} \mathrm{C}_{\text {org. }}$.

\section{INTRODUÇÃO}

Práticas agrícolas que objetivam menor degradação do solo e maior sustentabilidade da agricultura têm recebido atenção crescente, tanto por parte dos pesquisadores como dos agricultores. Neste contexto, o sistema de semeadura direta sem o preparo e revolvimento do sol o, o chamado plantio direto, a sucessão e a rotação de culturas têm sido bastante estudados, visto que tais práticas alteram, sensivelmente, os componentes físicos, químicos e biológicos dos solos. Com relação aos componentes microbianos conhece-se pouco, apesar de já ter sido evidenciado queestas alterações ocorrem de manei ra significativa, tanto em condições de dima temperado (Carter, 1986; Follet \& Schimel, 1989; Saffigna et al., 1989; I nsam et al., 1991) como tropical (Cattelan \& Vidor, 1990; Andrade et al., 1995; Bal ota et al., 1995; Hungria et al., 1995; Bal ota, 1997a,b ).

Os microrganismos do solo atuam nos processos de decomposi ção da matéria orgânica, participando diretamente no ciclo biogeoquímico dos nutrientes e, conseqüentemente, mediando a sua disponibilidade no solo. Assim, a biomassa microbiana total do solo funciona como importante reservatório de vários nutrientes das plantas (Grisi \& Gray, 1986), pois pertence ao componente lábil da matéria orgânica do solo, e possui atividade influenciada pelas condições bióticas e abióticas, o que permite que o seu acompanhamento reflita possíveis modificações no solo, podendo ser considerada como uma boa indicadora das alterações resultantes do manejo do solo. Há relatos, inclusive, de que essas mudanças nas propriedades microbiológicas causadas pelo preparo do solo e sucessão de culturas podem ser detectadas anteriormente às mudanças nos teores de C e N total do solo (Powlson \& J enkinson, 1981; Carter, 1986; Powlson et al., 1987; Saffigna et al., 1989; Andrade et al., 1995; Balota, 1997a,b). Nestecontexto, a biomassa microbiana (BM) pode ser utilizada para indicar o nível de degradação do solo (Hart et al., 1989; Smith \& Paul, 1990; Doran \& Parkin, 1994).

O objetivo destetrabal ho foi avaliar as al terações na atividade microbiana, a partir das estimativas do carbono e nitrogênio da bi omassa mi crobiana, em sol o submetido às sucessões de culturas trigo/soja e trigo/milho, em preparo do solo convencional e plantio direto.

\section{MATERIAL E MÉTODOS}

\section{Caracterização do experimento}

Utilizou-se um experimento de campo real izado, desde 1976, na Estação Experimental do Instituto Agronômico do Paraná-IAPAR, em um Latossolo 
Roxo distrófico em Londrina (PR), onde estavam sendo avaliados diferentes sistemas de preparo do solo e sucessão de culturas. Foram coletadas amostras nos seguintes tratamentos de sucessão de culturas: trigo/soja (T/S) e trigo/milho (T/M), submetidos ao preparo do solo convencional (PC) e em plantio direto (PD). O delineamento experimental de campo foi em parcelas subdivididas, em blocos casualizados, com três repetições. Os tratamentos de sucessão:T/S eT/M, constituíram as subparcelas, e os de preparo do solo convencional e direto as parcelas. Cada subparcela media 8 m delargura por $25 \mathrm{~m}$ de comprimento. Foram coletadas cinco subamostras, por subparcela, na profundidade de 0$15 \mathrm{~cm}$, dez dias após o plantio e sete dias antes da col heita das culturas de verão e de inverno dos anos de 1992 a 1994, ou seja, inverno de 92, 93 e 94 e verão de 92/93, 93/94 e 94/95. Após as amostragens no campo, dentro de um período de no máximo 8 horas, as amostras foram passadas em peneira de malha de $4 \mathrm{~mm}$, retirando-se subamostras para determinação da umidade em base gravimétrica e análise química e microbiológica.

\section{Análises laboratoriais}

Análise química: subamostras de solo foram secas ao ar, passadas em peneira de malha de $2 \mathrm{~mm}$, moídas e analisados o pH, a matéria orgânica (MO) e os teores de $\mathrm{P}, \mathrm{Ca}, \mathrm{Mg}, \mathrm{K}$ eAl, segundo Pavan et al. (1992).

Carbono da biomassa microbiana (C microbiano): utilizou-se o método de fumigaçãoincubação modificado de J enkinson \& Powlson (1976). Após a fumigação ou não do solo com clorofórmio isento de ál cool, o sol o foi incubado por três dias, à temperatura de $25^{\circ} \mathrm{C} . \mathrm{O} \mathrm{CO}_{2}$ liberado foi captado em uma solução que continha $10 \mathrm{~g} \mathrm{~L}^{-1}$ de $\mathrm{NaOH}$ equantificado no sistema FIA (Flow I njection Analysis), por diferenças na condutivimetria elétrica da solução. Para calcular o carbono da biomassa microbiana, utilizaram-se os valores obtidos nas amostras fumigadas e não fumigadas, com um $\mathrm{k}_{\mathrm{C}}$ de 0,45 (J enkinson \& Ladd, 1981).

O período de incubação de três dias foi definido baseado em ajustes metodológicos de preparo e incubação do solo e das variáveis do sistema FIA (Miyazawa et al., 1993), apresentando alto coeficiente de correlação $\left(r=0,81^{* *}\right)$ com o método original descrito por J enkinson \& Powlson (1976). Tal ajuste permitiu obter os dados com menor coeficiente de variação, em menor tempo de incubação e maior rapidez na determinação do $\mathrm{CO}_{2}$.

Nitrogênio da biomassa microbiana (N microbiano): o nitrogênio da biomassa microbiana foi avaliado segundo método de Brookes et al. (1985). Após a fumigação ou não do solo com clorofórmio isento de álcool, a extração de $\mathrm{N}$ das amostras foi feita com uma solução com 43,6 g L-1 de $\mathrm{K}_{2} \mathrm{SO}_{4}$, na proporção de 1:4 (solo:solução; massa:volume), sob agitação, a $175 \mathrm{rpm}$, por 60 minutos, centrifugação por 10 minutos a $2.500 \mathrm{rpm}$ e filtração em papel qualitativo, congel ando-se os extratos. A determinação do nitrogênio foi feita por col orimetria, segundo Keeney \& Nelson (1982). O N-microbiano foi cal culado pela equação $\mathrm{N}$-microbiano $=\mathrm{EN} / 0,54$, em que $\mathrm{EN}$ é a diferença entreo $\mathrm{N}$ extraído das amostras fumigadas e das não fumigadas (Brookes et al., 1985).

Respiração basal: valor obtido da medição de $\mathrm{CO}_{2}$ emanado das amostras não fumigadas durante o periodo de incubação, refletindo a atividade microbiana.

Quociente metabólico $\left(\mathbf{q} \mathrm{CO}_{2}\right)$ : representa a quantidade de $\mathrm{CO}_{2}$ liberado por unidade de bi omassa microbiana em determinado tempo. A obtenção dos dados foi feita pela divisão da respiração basal pelo carbono microbiano ( $\mu \mathrm{g} \mathrm{CO}_{2} / \mu \mathrm{g} \mathrm{C}_{\text {mic }} \mathrm{h}^{-1}$ ).

Todas as determinações foram feitas em triplicatas e os resultados expressos com base no solo seco. Os dados foram submetidos à análise de variância e feitas comparações das médias pel oTeste deTukey a $5 \%$.

\section{RESULTADOS E DISCUSSÃO}

Os dados da análise química (Quadro 1) evidenciam que práticas como o PD podem resultar num incremento de até $12 \%$ de matéria orgânica e $34 \%$ nos teores de P na camada superficial dos sol os (0-15 cm), como observado anteriormente por Saffigna et al. (1989), que constataram aumento gradual de até $7 \%$ no conteúdo de MO. Essas alterações, porém, geralmente estão restritas aos primeiros 5 a $10 \mathrm{~cm}$ do sol o (Sidiras \& Pavan, 1985; Campbel et al., 1989).

\section{Carbono e nitrogênio da biomassa mi crobiana}

Os valores do carbono da biomassa microbiana (CBM), desprendido como $\mathrm{C}-\mathrm{CO}_{2}$ em três dias de incubação, variaram de 84 a $595 \mu \mathrm{g} \mathrm{g}^{-1}$ e os de nitrogênio da biomassa microbiana (NBM), de 20 a $67 \mu \mathrm{g} \mathrm{g}^{-1}$, nas diferentes épocas de amostragens (Quadro 2). O menor valor foi observado na época decol heita de inverno sob o PC e o mai or na col heita de verão sob PD. Os val ores de CBM e NBM obtidos estão na mesma faixa aos já observados anteriormente por outros autores, sob condições edafoclimáticas diversas (Follet \& Schimel, 1989; Insam, 1990; Insam et al., 1991; Rodrigues et al., 1994; Alvarez et al., 1995; Andrade et al., 1995).

Os valores obtidos (Quadro 2) evidenciam que houve pequenas diferenças entre as sucessões de culturas, enquanto o preparo do solo influenciou a biomassa em todas as sucessões e épocas, tendo a 
Quadro 1. Análise química do solo(1) na camada de 0-15 cm de profundidade após vinte anos do cultivo contínuo de diferentes sucessões de culturas (T/S e T/M) e preparo do solo (convencional e direto)

\begin{tabular}{|c|c|c|c|c|c|c|c|c|}
\hline Tratamento & pH & MO & $\mathbf{P}$ & $\mathrm{Ca}$ & Mg & K & Al & $\mathbf{v}$ \\
\hline & & $\mathrm{g} \mathrm{kg}^{-1}$ & $\mathrm{mg} \mathrm{kg}^{-1}$ & 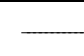 & $\mathrm{mol}_{\mathrm{c}} \mathrm{k}$ & - & 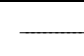 & 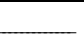 \\
\hline \multicolumn{9}{|l|}{ Convencional } \\
\hline $\begin{array}{l}\text { Trigo/soja } \\
\text { Trigo/milho }\end{array}$ & $\begin{array}{l}4,69 \\
4,57\end{array}$ & $\begin{array}{l}24,04 \\
25,90\end{array}$ & $\begin{array}{l}16,07 \\
15,76\end{array}$ & $\begin{array}{l}4,37 \\
4,02\end{array}$ & $\begin{array}{l}1,91 \\
1,76\end{array}$ & $\begin{array}{l}0,48 \\
0,49\end{array}$ & $\begin{array}{l}1,78 \\
2,94\end{array}$ & $\begin{array}{l}53,04 \\
49,21\end{array}$ \\
\hline \multicolumn{9}{|l|}{ Direto } \\
\hline $\begin{array}{l}\text { Trigo/soja } \\
\text { Trigo/milho }\end{array}$ & $\begin{array}{l}4,71 \\
4,57\end{array}$ & $\begin{array}{l}28,00 \\
28,40\end{array}$ & $\begin{array}{l}20,70 \\
21,85\end{array}$ & $\begin{array}{l}4,64 \\
4,40\end{array}$ & $\begin{array}{l}2,27 \\
1,99\end{array}$ & $\begin{array}{l}0,48 \\
0,51\end{array}$ & $\begin{array}{l}1,43 \\
4,16\end{array}$ & $\begin{array}{l}53,30 \\
49,18\end{array}$ \\
\hline
\end{tabular}

Quadro 2. Carbono e nitrogênio da biomassa microbiana e respiração basal na camada de 0-15 cm de profundidade no plantio e colhei ta das culturas de inverno e verão de 1992 a 1994, conforme a sucessão de culturas (T/S e T/M) e o preparo do solo (convencional e direto)

\begin{tabular}{|c|c|c|c|c|c|c|}
\hline \multirow{2}{*}{ Preparo do solo } & \multicolumn{2}{|c|}{ C da biomassa microbiana } & \multicolumn{2}{|c|}{ N da biomassa microbiana } & \multicolumn{2}{|c|}{ Respiração basal } \\
\hline & $\mathbf{P C}^{(1)}$ & $\mathbf{P D}^{(2)}$ & PC & PD & PC & PD \\
\hline & \multicolumn{4}{|c|}{$\mu g^{-1}$ de solo seco } & \multicolumn{2}{|c|}{$\mu \mathrm{g} \mathrm{g}^{-1} \mathrm{dia}^{-1}$ de $\mathrm{C}-\mathrm{CO}_{2}$ no solo } \\
\hline & \multicolumn{6}{|c|}{ Plantio de inverno } \\
\hline \multirow[t]{2}{*}{$\begin{array}{l}\text { Trigo/soja } \\
\text { Trigo/milho }\end{array}$} & $\begin{array}{l}112,94 b^{(3)} \\
304,81 a\end{array}$ & $\begin{array}{l}255,28 a \\
261,47 a\end{array}$ & $\begin{array}{l}31,04 b \\
40,44 b\end{array}$ & $\begin{array}{l}67,24 a \\
62,32 a\end{array}$ & $\begin{array}{l}1,85 b \\
2,43 a\end{array}$ & $\begin{array}{l}4,43 a \\
3,43 a\end{array}$ \\
\hline & \multicolumn{6}{|c|}{ Colheita de inverno } \\
\hline \multirow[t]{2}{*}{$\begin{array}{l}\text { Trigo/soja } \\
\text { Trigo/milho }\end{array}$} & $\begin{array}{r}83,90 b \\
144,62 a\end{array}$ & $\begin{array}{l}219,66 a \\
212,31 a\end{array}$ & $\begin{array}{l}32,47 b \\
32,62^{a}\end{array}$ & $\begin{array}{l}67,00 a \\
55,25 a\end{array}$ & $\begin{array}{l}2,44 a \\
2,66 b\end{array}$ & $\begin{array}{l}3,57 a \\
4,17 a\end{array}$ \\
\hline & \multicolumn{6}{|c|}{ Plantio de verão } \\
\hline \multirow[t]{2}{*}{$\begin{array}{l}\text { Trigo/soja } \\
\text { Trigo/milho }\end{array}$} & $\begin{array}{l}92,40 \mathrm{~b} \\
86,79 a\end{array}$ & $\begin{array}{l}240,45 a \\
203,94 a\end{array}$ & $\begin{array}{l}24,22 \mathrm{~b} \\
28,06 \mathrm{~b}\end{array}$ & $\begin{array}{l}47,11 a \\
64,72 a\end{array}$ & $\begin{array}{l}3,41 b \\
3,32 a\end{array}$ & $\begin{array}{l}5,37 a \\
3,94 a\end{array}$ \\
\hline & \multicolumn{6}{|c|}{ Colheita de verão } \\
\hline $\begin{array}{l}\text { Trigo/soja } \\
\text { Trigo/milho }\end{array}$ & $\begin{array}{l}166,20 \mathrm{~b} \\
179,01 \mathrm{~b}\end{array}$ & $\begin{array}{l}564,43 a \\
594,93 a\end{array}$ & $\begin{array}{l}29,15 \mathrm{~b} \\
19,66 \mathrm{~b}\end{array}$ & $\begin{array}{l}48,28 a \\
40,87 a\end{array}$ & $\begin{array}{l}2,98 b \\
3,01 b\end{array}$ & $\begin{array}{l}7,54 a \\
5,90 a\end{array}$ \\
\hline Média geral & $146,33 b$ & $319,06 a$ & $29,71 b$ & $56,60 a$ & $2,76 \mathrm{~b}$ & $4,79 a$ \\
\hline
\end{tabular}

(1) PC: Plantio convencional; (2)PD: Plantio direto. ${ }^{(3)}$ Médias seguidas da mesma letra, dentro da sucessão e da mesma época, não diferem estatisticamente pelo teste de Tukey a $5 \%$.

prática do PD proporcionado maiores val ores do que a do PC. O PD apresentou acréscimos na CBM, em relação ao PC, de 24, 89, 148 e 236\%, respectivamente, na época de plantio e col heita de inverno e verão, e os acréscimos na NBM variaram de 81 a 114\%. A sucessão de cultura teve efeito apenas na CBM no PC do plantio de inverno e na NBM no PD do plantio de verão, quando o T/M foi superior ao T/S, respectivamente, em 170 e 37\% (anál ises não apresentadas). Os valores médios totais de PD foram 118 e $101 \%$ maiores que os de PC para CBM e NBM, respectivamente.
O efeito de diferentes tipos de preparo do solo na biomassa microbiana já havia sido observado por Follet \& Schimel (1989) em experimento desenvol vido por 16 anos a oeste de Nebraska, EUA, em que os valores de CBM e NBM foram,

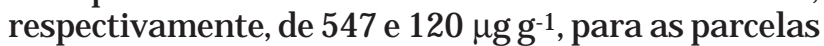
de PC, e de 864 e $175 \mu \mathrm{g} \mathrm{g}^{-1}$, para aquelas sob PD. Esses autores observaram que a prática de PD incrementou a CBM em 58\% em avaliação feita aos 10 dias eem $72 \%$ aos 36 dias de incubação, enquanto, para os valores de NBM, esses incrementos foram de, respectivamente, 46 e 62\% em relação à prática 
do PC. Convém salientar, porém, que, mesmo nas parcelas sob PD, os valores obtidos foram inferiores em $175 \%$ para CBM e $139 \%$ para NBM, em 10 dias de incubação, aos das parcelas com gramíneas nativas que nunca foram submetidas ao cultivo, segundo Follet \& Schimel (1989).

Alvarez et al. (1995), estudando sol os típicos dos pampas argentinos, com 12 anos de cultivo sob PC e $\mathrm{PD}$, observaram que a prática do PD resultou num incremento de $50 \%$ no teor dematéria orgânica (MO) nos primeiros $5 \mathrm{~cm}$ do sol o e de até $8 \%$ nos primeiros $20 \mathrm{~cm}$ do solo. O PD aumentou em quase três vezes a CBM em relação às parcelas sob PC (aumento de 160 para $450 \mu \mathrm{g} \mathrm{g}^{-1}$ ). Outros trabal hos desenvolvidos em solo do norte e sudoeste do Paraná têm evidenciado, de maneira sistemática, que a prática doPD proporciona aumento na biomassa microbiana nas diversas condições de manejo das culturas (Balota, 1997a,b).

\section{Respiração basal}

As amostras, tanto do plantio como da col heita de inverno, apresentaram menor atividade respiratória (1,85 a 4,37 $\mathrm{gg} \mathrm{g}^{-1}$ dia-1 de $\mathrm{C}-\mathrm{CO}_{2}$ no solo) queas coletadas no período do verão $\left(2,98\right.$ a $7,54 \mu \mathrm{g} \mathrm{g}^{-1} \mathrm{dia}^{-1}$ de $\mathrm{C}-\mathrm{CO}_{2}$ no solo) (Quadro 2). N ão houve diferenças estatísticas na respiração basal, conforme a sucessão de culturas (análises não mostradas), ao passo que o PD teve maior atividade respiratória em todas as sucessões e épocas amostradas que o PC (41 e 139\% no inverno e 57 e $96 \%$ no verão). O val or médio total de incremento da respiração basal obtido pelo PD foi $73 \%$ maior que o do PC.

Contudo, vale destacar que os valores de respiração basal descritos pela literatura são bastante variados. Insam et al. (1991), analisando amostras provenientes de solos cultivados com a mesma cultura por até 77 anos, observaram que a respiração basal variou de 8,40 a 33,60 $\mu \mathrm{g} \mathrm{g}^{-1}$ dia-1 de $\mathrm{CO}_{2}$ no solo. Rodrigues et al. (1994), por sua vez, constataram que a respiração basal, em sol os coletados sob condição tropical, variou de 6,3 a $20 \mu \mathrm{g} \mathrm{g}^{-1}$ dia-1 $^{-1}$ de $\mathrm{C}-\mathrm{CO}_{2}$ no solo, enquanto que nos pampas argentinos, a variação foi de 3,3 a $34,4 \mu \mathrm{g} \mathrm{g}^{-1}$ dia-1 de $\mathrm{C}-\mathrm{CO}_{2}$ no solo (Alvarez et al., 1995). Nestes experimentos, deve-se considerar a grande variabilidade de sol o e clima, bem como as condições metodológicas analíticas.

A mai or liberação de $\mathrm{CO}_{2}$ observada em parcelas de PD, comparadas ao PC, é devida à mai or atividade biológica (Carter, 1986; Follet \& Schimel, 1989) que, por sua vez, está relacionada diretamente com o C do solo e, ou, da biomassa microbiana (Hendrix et al., 1988). Follet \& Schimel (1989), em uma área submetida ao PD por 16 anos, constataram um incremento de $108 \%$ na li beração de $\mathrm{CO}_{2}$ do sol o com 36 dias de incubação, em relação às parcelas com preparo do solo, enquanto Alvarez et al. (1995) observaram aumento de $220 \%$ para oPD em relação às parcelas com preparo (de 10 para $32 \mu \mathrm{g} \mathrm{g}^{-1} \mathrm{dia}^{-1}$ de $\mathrm{C}-\mathrm{CO}_{2}$ no solo). Entretanto, Saffigna et al. (1989) observaram di mi nuição da respiração específica com a prática do PD e, embora não tenham conseguido explicar o ocorrido, atribuíram o fato a diferenças na decomposição da MO ou atividade da biomassa microbiana.

\section{Quociente metabólico (respiração basal/ $\mathbf{C}_{\text {mic }}$ )}

Os valores do quociente metabólico variaram de 3,32 a 15,90, para o PC, e de 4,13 a 9,31, para o PD (Quadro 3). Não foram observadas diferenças significativas entreas sucessões T/S eT/M, tanto no PC como no PD (análises não mostradas), ao passo que o preparo do solo influenciou o quociente metabólico em todas as sucessões e épocas de amostragens. De modo geral, a prática do PD proporcionou menor quociente metabólico que o apresentado pelo PC, nas duas sucessões em todas as amostragens. O valor médio total do PD foi $28 \%$ inferior ao do PC.

Alvarez et al. (1995) observaram que parcelas submetidas ao PD apresentaram valores maiores de $\mathrm{qCO}_{2}$ que parcelas com PC, com acréscimos de $60 \%$ (de 0,050 para $0,080 \mu \mathrm{g}$ de $\mathrm{C}-\mathrm{CO}_{2} \mu \mathrm{g}^{-1}$ de $\mathrm{CBM}$ ) na profundidade de $5 \mathrm{~cm}$, enquanto, com $15 \mathrm{~cm}$, ocorreu

Quadro 3. Quociente metabólico $\left(\mathrm{qCO}_{2}\right)$ e relação $\mathrm{C}_{\text {mid }} / \mathrm{C}_{\text {org }}$ na camada de $0-15 \mathrm{~cm}$ de profundidade no plantio e colheita das culturas de inverno e verão de 1992 a 1994, conforme a sucessão de culturas (T/S e T/M) e o preparo do solo (convencional e direto)

\begin{tabular}{|c|c|c|c|c|}
\hline \multirow{2}{*}{ Preparo do solo } & \multicolumn{2}{|c|}{ Quociente metabólico $^{(\mathbf{1})}$} & \multicolumn{2}{|c|}{$C_{\min } / C_{\text {org }}{ }^{(2)}$} \\
\hline & $P C^{(3)}$ & PD ${ }^{(4)}$ & PC & PD \\
\hline & \multicolumn{2}{|c|}{$\left(\mu \mathrm{gCO}_{2} / \mu \mathrm{gC}_{\min } \mathrm{h}^{-1}\right) \times 10^{-4}$} & \multicolumn{2}{|c|}{$\%$} \\
\hline & \multicolumn{4}{|c|}{ Plantio de inverno } \\
\hline \multirow[t]{2}{*}{$\begin{array}{l}\text { Trigo/soja } \\
\text { Trigo/milho }\end{array}$} & $\begin{array}{l}6,82 a^{(5)} \\
3,32 a^{2}\end{array}$ & $\begin{array}{l}7,14 a \\
5,47 a\end{array}$ & $\begin{array}{l}0,81 a^{(3)} \\
2,09 a\end{array}$ & $\begin{array}{l}1,58 a \\
1,60 a\end{array}$ \\
\hline & \multicolumn{4}{|c|}{ Colheita de inverno } \\
\hline \multirow[t]{2}{*}{$\begin{array}{l}\text { Trigo/soja } \\
\text { Trigo/milho }\end{array}$} & $\begin{array}{r}12,10 a \\
7,67 a\end{array}$ & $\begin{array}{l}6,76 b \\
8,18 a\end{array}$ & $\begin{array}{l}0,60 \mathrm{~b} \\
0,96 \mathrm{a}\end{array}$ & $\begin{array}{l}1,36 a \\
1,32 a\end{array}$ \\
\hline & \multicolumn{4}{|c|}{ Plantio de verão } \\
\hline \multirow[t]{2}{*}{$\begin{array}{l}\text { Trigo/soja } \\
\text { Trigo/milho }\end{array}$} & $\begin{array}{l}15,40 a \\
15,90 a\end{array}$ & $\begin{array}{l}9,31 b \\
8,06 b\end{array}$ & $\begin{array}{l}0,65 b \\
0,58 b\end{array}$ & $\begin{array}{l}1,49 a \\
1,24 a\end{array}$ \\
\hline & \multicolumn{4}{|c|}{ Colheita de verão } \\
\hline $\begin{array}{l}\text { Trigo/soja } \\
\text { Trigo/milho }\end{array}$ & $\begin{array}{l}7,47 a \\
7,00 a\end{array}$ & $\begin{array}{l}5,57 a \\
4,13 b\end{array}$ & $\begin{array}{l}1,18 b \\
1,22 b\end{array}$ & $\begin{array}{l}3,50 a \\
3,65 a\end{array}$ \\
\hline Média geral & $9,46 a$ & $6,83 b$ & $1,01 b$ & $1,97 a$ \\
\hline
\end{tabular}


o inverso, isto é, o PD foi inferior ao PC em cerca de $21 \%$. Insan et al. (1991) verificaram que tanto o preparo como o manejo do solo al teram o $\mathrm{qCO}_{2}$, em experimentos submetidos ao mesmo tratamento por 77 anos. Nesses tratamentos, as parcelas que receberam quantidade de fertilizantes reduzida ou limitada apresentaram mai or $\mathrm{qCO}_{2}$, comparadas às que receberam quantidade adequada de fertilizantes.

Para Insam \& Domsch (1988), a respiração microbiana por unidade de biomassa microbiana diminui em sistemas mais estáveis. Por outro lado, a incorporação de resíduos de culturas ao solo aumenta o quociente metabólico (Ocio \& Brookes, 1990). Assim, parece ocorrer uma relação inversa entre a biomassa microbiana e o quociente metabólico, sugerindo que, em maiores teores de C, podem ocorrer aumento da BM e diminuição na atividade metabólica (I nsam et al., 1991), como também foi constatado neste trabalho.

Para Saffigna et al. (1989), solos com preparo convencional podem apresentar menor conteúdo de MO e uma BM com mai or quociente metaból ico que aquelas sob PD. I sto pode ocorrer pela diferença de acessibilidade do substrato de $\mathrm{C}$ aos microrganismos e, ou, mudanças nos padrões metabólicos ou, ainda, al terações na composição da microbiota (Alvarez et al., 1995). O PD pode, por exemplo, proporcionar maior agregação das partículas do solo, onde os microrganismos podem-se proteger de condições adversas. A comunidade microbiana, tornando-se mais estável, resultaria numa diferenciação de acordo com sua idade como "nova" e"vel ha". Células "novas" são metabolicamente mais ativas, refl etindo maior $\mathrm{qCO}_{2}$. I sto tem implicações práticas importantes na agricultura, pois se mais $C$ é respirado, maior quantidade de $C$ pode estar sendo liberado do solo, acarretando diminuição dos teores de C no solo (Insam et al., 1991). Entretanto, não deve ser esquecido que o tipo de manejo do sol o pode sel ecionar uma população microbiana mais eficiente, que perderia menos C via respiração. Essa característica da comunidade microbiana pode ser muito importante na manutenção dos teores de C do solo (Insam, 1990).

\section{Relação carbono da biomassa microbiana: carbono orgânico total ( $\left.\mathrm{C}_{\mathrm{MIC}} / \mathrm{C}_{\mathrm{ORG}}\right)$}

Não houve grandes diferenças nos valores da relação CBM e o C orgânico total do solo, em função da sucessão de culturas, enquanto, em relação ao preparo do sol o, os valores variaram de 0,58 a 2,09\%, para o PC, e de 1,24 a 3,65\%, para o PD (Quadro 3). O PD apresentou maiores val ores queo PC em todas as sucessões eem todas as amostragens, com exceção doT/M no plantio de inverno. Com relação à sucessão de culturas, foi observada diferença significativa apenas na T/M em relação ao T/S no PC do plantio de inverno, com acréscimos de 158\% (análises não mostradas).
Os valores médios observados para cada época amostrada indicam que PD apresentou acréscimos em relação ao PC de 10, 72, 111 e 176\%, respectivamente, nas amostragens do plantio e colheita de inverno e verão. Na média geral, as parcelas com PD apresentaram relação $C_{\text {mid }}$ ( $C_{\text {org }} 95 \%$ superior à apresentada pelas parcelas com PC.

Carter (1986) constatou que o preparo do solo resulta em diminuição do $C_{\text {mic }}$ e da relação $C_{\text {mid }} C_{\text {org. }}$. Insam (1990), estudandoáreas com várias culturas, observaram variação de 0,6 a 3,74\% na relação $\mathrm{C}_{\text {mid }} / \mathrm{C}_{\text {org, }}$ e Andrade et al. (1995), num experimento de cafeeiro, observaram quea relação $\mathrm{C}_{\text {mid }} \mathrm{C}_{\text {org }}$ variou de 1,7 a 8,9\%, conformea apl icação de cal cário. Essas variações podem estar relacionadas com o manejo do solo, como monocultura e rotação, que apresentaram, respectivamente, val ores de 1,8 e2,25\% (I nsam et al., 1989) ou 2,3 e 2,9\% (Anderson \& Domsch, 1989). E ntretanto, para outros autores, essas relações estariam entre 1,8 e 2,1\%, para parcelas cultivadas com monoculturas permanentes (Brookes et al., 1984), entre 2,5 e 3,3\%, para áreas com rotação de culturas permanentes (M cGill et al., 1986).

A biomassa microbiana representa de 1 a 3\% (Powlson \& J enkinson, 1981) ou até 5\% (Sparling, 1992) do C orgânico total do solo. Contudo, a relação $\mathrm{C}_{\text {mid }} / \mathrm{C}_{\text {org }}$ abrange amplo espectro, variando de 0,27 a $7,0 \%$, segundo revisão de Anderson \& Domsch (1989). Esse amplo espectro é devido às diferenças de tipo e manejo do solo, de épocas de amostragem e de métodos analíticos utilizados.

As mudanças na relação $C_{\text {mid }} / C_{\text {org }}$ refletem o padrão de entrada de $M O$ nestes solos, a eficiência da conversão do $\mathrm{C}$ microbiano, as perdas do $\mathrm{C}$ do solo e a estabilização do C orgânico pela fração mineral do solo (Sparling, 1992). O nível da relação $\mathrm{C}_{\text {mid }} / \mathrm{C}_{\text {org }}$ podeindicar se o carbono está em equilíbrio, se está acumulando ou diminuindo (Anderson \& Domsch, 1989; I nsam, 1990).

O C do solo é composto da parte lábil e da parte resistente ou recalcitrante ao ataque de microrganismos (Alvarez et al., 1995). O CBM, sendo parte do C orgânico lábil, atua diretamente no ciclo do $C$, podendo responder mais rapidamente a mudanças nos padrões de entrada ou níveis de decomposição da MO que a MO como um todo (Powlson et al., 1987).

A relação $\mathrm{C}_{\text {mic }} / \mathrm{C}_{\text {org }}$, também denominada de "quociente microbiano", tem sido considerada como boa indicadora das alterações dos processos no solo (Anderson \& Domsch, 1989). Solos que exibem valores maiores ou menores poderiam expressar a ocorrência, respectivamente, de acúmulo ou perda de C do solo. J enkinson \& Ladd (1981), por sua vez, citam 2,2\% como sendo o nível no qual estaria ocorrendo equilíbrio em parcelas cultivadas.

Assim, a relação $\mathrm{C}_{\text {mid }} / \mathrm{C}_{\text {org }}$ parece ser boa indicadora das alterações na MO em função do manejo do sol o (Carter, 1986; I nsam \& Domsch, 1988; 
Insam, 1990; Insam et al., 1991; Sparling, 1992; Alvarez et al., 1995; Andradeet al., 1995). Entretanto, tais relações podem necessitar de interpretações locais, em virtude dos vários fatores ambientais que as estariam influenciando (Sparling, 1992).

Houve correlação linear positiva e significativa entre o $\mathrm{C}$ da biomassa microbiana e o C orgânico total $\left(r=0,88^{* *}\right)$, e o $\mathrm{N}$ da biomassa microbiana de $N\left(r=0,86^{* *}\right)$ (Figura 1$)$ e entre a respiração basal eoCBM ( $\left.r=0,81^{* *}\right)$ ea BMN ( $\left.r=0,83^{* *}\right)$, como pode ser observado na figura 2. Correlação significativa entre os parâmetros microbiológicos e a produção das culturas têm sido observadas em vários trabalhos. Como exemplo, avaliando 134 parcelas em 25 experimentos em condições de clima temperado na zona central da Europa, Anderson \& Domsch (1989) observaram alta correlação significativa entre a CBM e o C orgânico do solo, tanto em rotação de culturas $(r=0,97)$ como em monoculturas $(r=0,89)$, enquanto Carter (1986) observou correlação menor $(r=0,68)$. Em estudo similar ao desenvolvido por Anderson \& Domsch (1989), I nsam et al . (1989), nos EUA, encontraram correlação de $r=0,34$ para
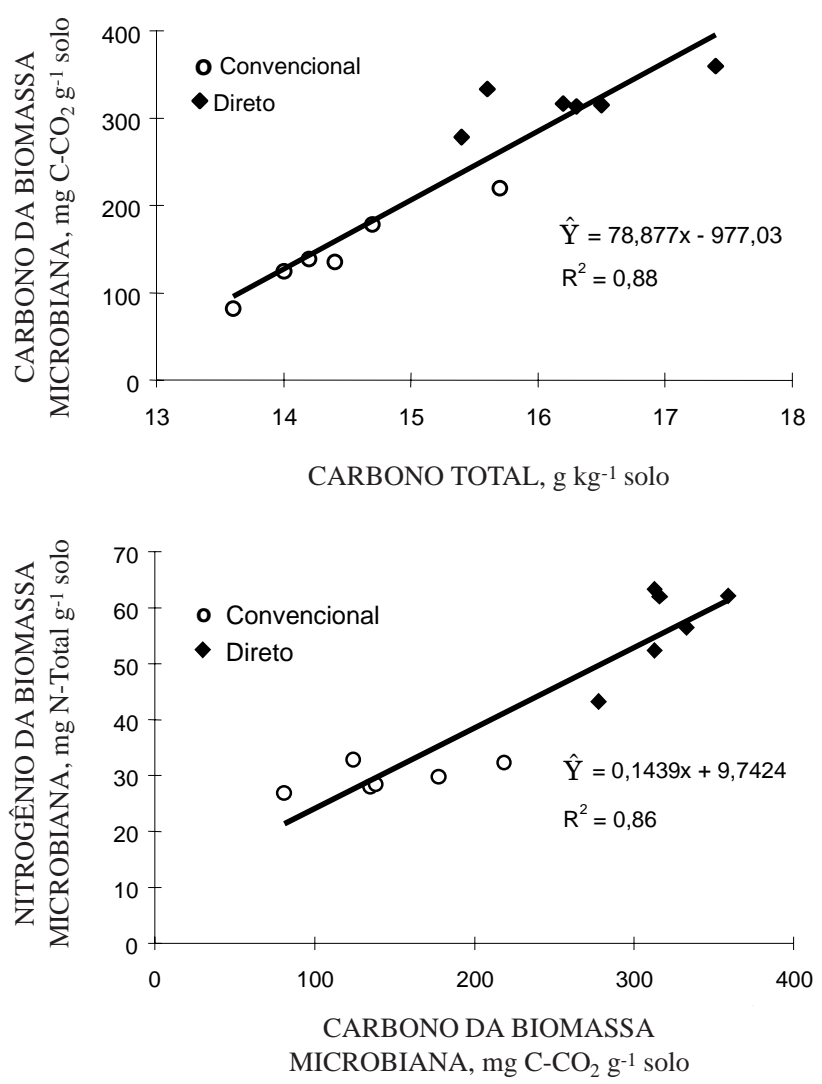

Figura 1. Regressão entre o carbono da biomassa microbiana e o carbono total e entre o nitrogênio da biomassa microbiana e o carbono da bi omassa microbiana (preparo convencional e plantio direto) para 12 dados a $0,1 \%$.
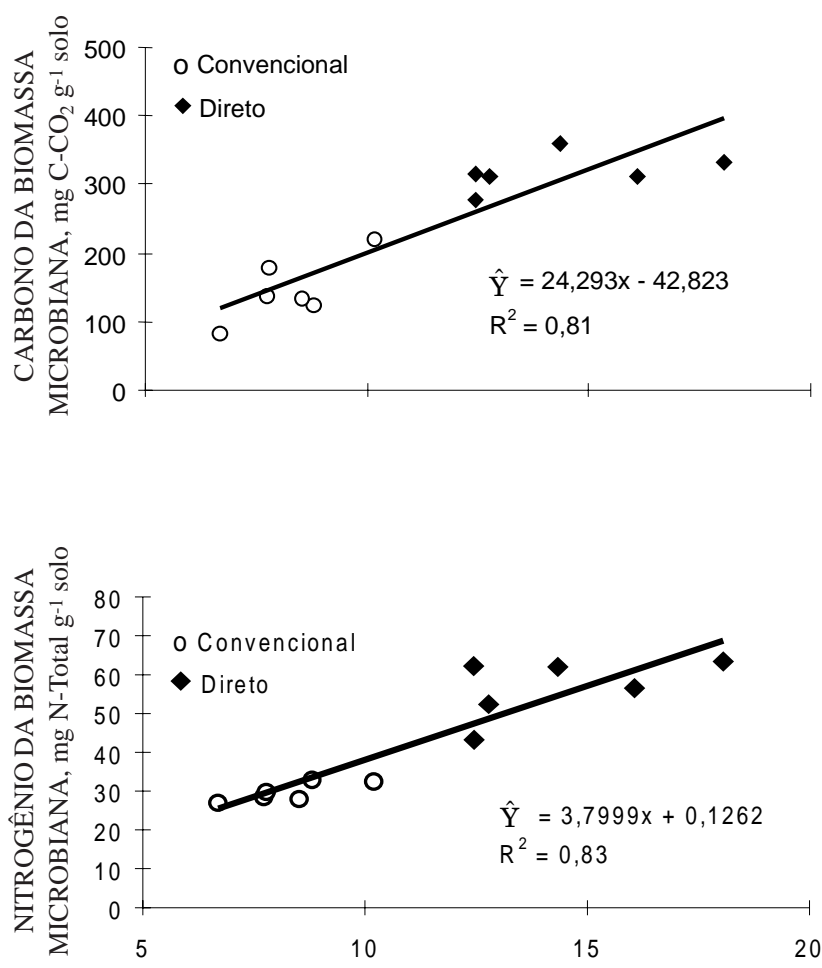

RESPIRAÇÃO BASAL, mg C-CO $\mathrm{C}^{-1}$ solo

Figura 2. Regressão entre a respiração basal e o carbono da biomassa microbiana e entre o nitrogênio da biomassa microbiana (preparo convencional e plantio direto) para 12 dados a $0,1 \%$.

rotação e $r=0,50$ para monocultura. Insam et al. (1991), por sua vez, obtiveram alta correlação positiva da $\mathrm{CBM}$ e a relação $\mathrm{C}_{\text {mid }} / \mathrm{C}_{\text {org }}$ e a produção degrãos ( $r=0,77$ e 0,71 , respectivamente) enegativa entre o $\mathrm{qCO}_{2}$ e a produção de grãos $(r=-0,78)$, não havendo correlação entre a respiração basal e a produção de grãos.

Os acréscimos da biomassa microbiana em parcelas submetidas ao PD são devidos ao fato de tal prática proporcionar condições mais favoráveis ao desenvolvimento de microrganismos, como, por exemplo, temperatura, umidade, aeração, etc, e menor taxa de decomposição de componentes orgânicos, além de O PD favorecer algumas propriedades químicas do solo, como $\mathrm{pH}$ e CTC, e os teores de $\mathrm{Ca}, \mathrm{Mg}, \mathrm{K}, \mathrm{P}$ (Sidiras \& Pavan, 1985). A prática do PD em solo LR argiloso, tanto do norte como sudoeste paranaense, tem proporcionado alterações no número de vários grupos de microrganismos benéficos (amonificadores, nitrificadores, bradyrhizobium, azospirillum, solubilizadores de fosfato e fungos micorrízicos arbusculares), bem como na atividade enzimática do solo (Hungria et al., 1995; Balota, 1997a,b). 
McGill et al. (1986) observaram que a disponibilidade de C é temporariamente maior em parcelas manejadas organicamente, porque estas parcelas contêm, significativamente, maior quantidade de $\mathrm{C}$ orgânico solúvel que parcelas que receberam fertilizantes inorgânicos. Esses compostos orgânicos incrementam a BM, em comparação aos fertilizantes inorgânicos, porque eles aumentam as proporções de C eN lábeis, estimulando diretamente a atividade da biomassa. N essas condições, pode-se estabelecer maior diversidade de decompositores com maior eficiência na utilização do substrato, resultando, assim, numa maior relação $\mathrm{C}_{\text {mid }} / \mathrm{C}_{\text {org }}$ (Insam, 1990).

Assim, o manejo do solo pode determinar al terações na BM mais detectáveis queas observadas na MO. N este contexto, a BMS pode ser considerada como boa indicadora das alterações ocorridas no sol o conforme seu manejo (Carter, 1986; Saffigna et al, 1989). Os dados obtidos no presente trabalho de incremento na biomassa microbiana, na respiração basal ena relação $C_{\text {mid }} / C_{\text {org }}$ e diminuição no quociente metabólico, determinado pela prática do PD, reforçam este argumento.

\section{CONCLUSÕES}

1. O plantio direto proporcionou maior biomassa microbiana de carbono e de nitrogênio, maior respiração basal e maior relação $\mathrm{C}_{\text {mid }} / \mathrm{C}_{\text {org }}$.

2. O plantio direto proporcionou diminuição no quociente metabólico $\left(\mathrm{qCO}_{2}\right)$, o que resultou em menor perda de carbono do solo.

3. A atividade da BM mostrou-se boa indicadora das alterações microbianas ocorridas no solo, conforme o manejo.

\section{AGRADECIMENTOS}

Ao Técnico Agrícola Aresdimundinei Dias Campos e aos Técnicos de Laboratório Maria A. Mattos e Oswaldo Machineski, pelo auxílio na execução do trabalho.

\section{LITERATURA CITADA}

ALVAREZ, R.; DÍAZ, R.A.; BARBERO, N.; SANTANATOGLIA, O.J . \& BLOTTA, L. Soil organic carbon, microbial biomass and $\mathrm{CO}_{2}-\mathrm{C}$ production from three tillage systems. Soil Till. Res., 33:17-28, 1995.

ANDERSON, T.-H. \& DOMSCH, K.H. Ratios of microbial biomass carbon to total organic carbon in arable soils. Soil Biol. Biochem., 21:471-479, 1989.
ANDRADE, D.S.; COLOZZI-FILHO, A.; PAVAN, M.A.; BALOTA, E.L. \& CHAVES, J.C.D. Atvidade microbiana em função da calagem em um solo cultivado com cafeeiro. R. Bras. Ci. Solo, 19:191-196, 1995.

BALOTA, E.L. Alterações microbiológicas em solo cultivado sob o plantio direto. In: PEIXOTO, R.T.G.; AHRENS, D.C. \& SAMAHA, M.J., eds. Plantio direto: caminho para uma agricultura sustentável. Ponta Grossa, IAPAR,PRP/PG, 1997a. p.222-233.

BALOTA, E.L. Atividade microbiana em solo sob plantio direto. In: CONFERÊNCIA ANUAL DE PLANTIO DIRETO, 2., Pato Branco. 1997. Resumos de Palestras. Passo Fundo, Aldeia Norte, 1997b. p.35-50.

BALOTA, E.L.; ANDRADE, D.S. \& COLOZZI-FILHO, A. Avaliações microbiológicas em sistemas de preparo do solo esucessão de culturas. In: CONGRESSO BRASILEIRO DE PLANTIO DIRETO PARA UMA AGRICULTURA SUSTENTÁVEL, 1., Ponta Grossa, 1995. Anais. Ponta Grossa, IAPAR, 1995. p.9-11

BROOKES, P.C.; POWLSON, D.S. \& JENKINSON, D.S. Phosphorus in the soil microbial biomass. Soil Biol. Biochem., 16:169-175, 1984.

BROOKES, P.C.; LANDMAN, A; PRUDEN, G. \& JENKINSON, D.S. Chloroform fumigation and the realease of soil nitrogen: a rapid direct extration method to measure microbial biomass nitrogen in soil. Soil Biol. Biochem., 17:837-842, 1985.

CAMPBEL, C.A.; BIERDERBECK, V.O.; SCHINITZER, M.; SELLES, F. \& ZENTNER, R.P. Effect of 6 years of zero tillage and $\mathrm{N}$ fertilizer management on change in soil quality of an orthic Brown Chernozen in southwestern Saskatchewan. Soil Till. Res., 14:39-52, 1989.

CARTER, M.R. Microbial biomass as an index for tillage-induced changes in soil biological properties. Soil Till. Res., 7:29-40, 1986.

CATTELAN, A.J . \& VIDOR, C. Flutuacões na biomassa, atividade e população microbiana do solo, em função de variações ambientais. R. Bras. Ci. Solo, 14:133-142, 1990.

DORAN, J.W. \& PARKIN, T.B. Defining and assessing soil quality. In: DORAN, J.W.; COLEMAN, D.C.; BEZDICEK, D.F. \& STEWART, B.A., eds. Defining soil quality for a sustainable environment. Madison, Soil Science Society of America, 1994. p.3-35.

FOLLET, R.F. \& SCHIMEL, D.S. Effect of tillage practices on microbial biomass dynamics. Soil Sci. Soc. Am. J ., 53:10911096, 1989.

GRISI, B.M. \& GRAY, T.R.G. Comparação dos métodos de fumigação, taxa de respiração em resposta à adição de glicose e conteúdo de ATP para estimar a biomassa microbiana do solo. R. Bras. Ci. Solo, 10:109-115, 1986.

HART, P.B.S.; AUGUST, J.A. \& WEST, A.W. Long-term consequences of topsoil mining on select biological and physical characteristics of two New Zealand loessial soils under grazes pasture. Land Degrad. Rehabil., 1:77-88, 1989. 
HENDRIX, P.F.; HAN, C.-R.; GROFFMAN, P.M. Soil respiration in conventional and no-tillage agroecosystems under different winter cover crop rotations. Soil Till. Res., 12:135$148,1988$.

HUNGRIA, M.;ANDRADE, D.S.; COLOZZI FILHO,A.;BALOTA, E.L. \& SANTOS, J.C. Ecologia microbiana em solos sob cultivo na região sul do Brasil. In: HUNGRIA, M.;BALOTA, E.L.; COLOZZI-FILHO, A. \& ANDRADE, D.S., eds. Microbiologia do solo: Desafios para o séculoXXI. Londrina, IAPAR/EMBRAPA-CNPSo, 1995. p.234-270.

INSAM, H. Are the soil microbial biomass and basal respiration governed by the climatic regime? Soil Biol. Biochem., 22:525-532, 1990.

INSAM, H. \& DOMSCH, K.H. Relationship between soil organic carbon and microbial biomass on chronosequences of reclamation sites. Microb. Ecol., 15:177-188, 1988.

INSAM, H.; MITCHELL, C.C. \& DORMAAR, J.F. Relationship of soil microbial biomass and activity with fertilization practice and crop yield of three ultisols. Soil Biol. Biochem., 23:459-464, 1991.

INSAM, H.; PARKINSON, D. \& DOMSCH, K.H. The influence of macroclimate on soil microbial biomass levels. Soil Biol. Biochem., 21:211-221, 1989.

J ENKINSON, D.S \& LADD, J.N. Microbial biomass in soil: Measurement and turnover. In: PAUL, E.A.\& LADD, J .M. eds. Soil biochemistry, vol.5. NewYork, Marcel Decker, 1981. p.415-471.

J ENKINSON, D.S. \& POWLSON, D.S. The effects of biocidal treatments on metabolism in soil.V-A method for measuring soil biomass.Soil Biol. Biochem., 8:209-213, 1976.

KEENEY, D.R. \& NELSON, D.W. Nitrogen. Inorganic forms. In: PAGE, A.L., ed. Method of soil analysis chemical and microbiological. 2.ed. Madison, American Society of Agronom, 1982. p.643-693.

McGILL, W.B.; CANNON, K.R.; ROBERTSON, J.A. \& COOK, F.D. Dynamics of soil microbial biomass and water-soluble organic $C$ in Breton $L$ after 50 years of cropping to two rotations. Can. J. Soil Sci., 66:1-19, 1986.
MIYAZAWA, M.; BALOTA, E.L.; ANDRADE, D.S.; PAVAN, M.A. \& COLOZZI-FILHO, A. Determinação de $\mathrm{CO}_{2}$ em análise por injeção em fluxo para estimar a biomassa microbiana do solo. In: CONGRESSO BRASILEIRO DE MICROBIOLOGIA, 17., Santos, 1993. Resumos. Santos, SBM, 1993. p.B3.013.

OCIO, J.A. \& BROOKES, P.C. An evaluation of methods for measuring the microbial biomass in soils following recent additions of wheat straw and characterization of the biomass that devel ops. Soil Biol. Biochem., 22:685-694, 1990.

PAVAN, M.A.; BLOCH, M.F.; ZEMPLSKI, H.C.; MIYAZAWA, M \& ZOCOLER, D.C. Manual de análise química de solo e controle de qualidade. Londrina, I APAR, 1992. 40p. (Circular Técnica, 76)

POWLSON, D.S. \& J ENKINSON, D.S. A comparison of the organic matter, biomass, adenosine triphosphate and mineralizable nitrogen contents of ploughed and directdrilled soils. J .Agric. Sci., 97:713-721, 1981.

POWLSON, D.S.; BROOKES, P.C. \& CHRISTENSEN, B.T. Measurement of soil microbial biomass provides an early indication of changes in the total soil organic matter due to straw incorporation. Soil Biol. Biochem., 19:159-164, 1987.

RODRIGUES, E.F.G.; GUERRA, J .G.N.; ALMEIDA, D.L. \& DEPOLLI, H. Biomassa microbiana de carbono de solos de Itaguaí (RJ ): comparação entre os métodos fumigaçãoincubação e fumigação-extração. R. Bras. Ci. Solo, 18:427432, 1994.

SAFFIGNA, P.G.; POWLSON, D.S.; BROOKES, P.C. \& THOMAS G.A. Influence of sorghum residues and tillage on soil organic matter and soil microbial biomass in an Australian Vertisol. Soil Biol. Biochem., 21:759-765, 1989.

SIDIRAS, N. \& PAVAN, M.A. Influência do sistema de manejo do solo no seu nível de fertilidade. R. Bras. Ci. Solo, 9:249254, 1985

SMITH,J.L. \& PAUL, E.A.The significance of microbial biomass estimations. In: BOLLAG, J.M \& STOZKY, G., eds. Soil biochemistry. New York, Marcel Decker, 1990. p.357-396.

SPARL ING, G.P. Ratio of microbial biomass carbon to soil organic carbon as a sensitive indicator of changes in soil organic matter. Aust. J. Soil Res., 30:195-207, 1992. 